\title{
Lessons Learned from Supplementing Archaeological Museum Exhibitions with Virtual Reality
}

\author{
Anna Puig • Inmaculada Rodríguez • \\ Josep Ll. Arcos • Juan A. \\ Rodríguez-Aguilar • Sergi Cebrián • \\ Anton Bogdanovych • Núria Morera . \\ Antoni Palomo • Raquel Piqué
}

Received: date / Accepted: date

\begin{abstract}
Archaeological excavations provide us with important clues about the past. Excavated artefacts represent an important connection to civilisations that no longer exist and help us understand some of their customs, traditions and common practices. With the help of academics and practitioners from various disciplines the results of archaeological excavations can be analysed and a body of knowledge about the corresponding society can be created and shared with members of the general public. Museums have traditionally served the purpose of communicating this knowledge and backing it up with the help of the excavated artefacts. Many museum visitors, however, find it difficult to develop a coherent understanding of the corresponding society only based on the artefacts and annotations showed in museums. Effective modern techniques that have high potential in helping museum visitors with better understanding of the past are 3D reconstruction and Virtual Reality. 3D reconstruction offers a cost effective way of recreating historical settlements in a computer-generated virtual environment, while Virtual Reality helps with immersing people into such environments and reaching a high degree of real-
\end{abstract}

\author{
Anna Puig, Inmaculada Rodríguez \\ University of Barcelona \\ E-mail: \{anna,inmarodriguez\}@ub.edu \\ Josep Ll. Arcos, Juan A. Rodríguez-Aguilar, Sergi Cebrián \\ IIIA-CSIC E-mail: \{arcos,jar,scebrian\}@iiia.csic.es \\ Anton Bogdanovych \\ University of Western Sydney \\ E-mail: A.Bogdanovych@westernsydney.edu.au \\ Núria Morera, Raquel Piqué \\ Autonomous University of Barcelona \\ E-mail: \{nuria.morera,raquel.pique\}@uab.cat \\ Antoni Palomo \\ Museu d'Arqueologia de Catalunya \\ E-mail: antoni.palomo@gencat.cat
}


ism. With the help of these technologies it becomes possible to relive history, imagine yourself being a part of the reconstructed society and learn about its culture firsthand. The combination of 3D reconstruction and Virtual Reality represents a very powerful learning tool, however this tool has been rarely used in a museum setting and its correct use has not been properly investigated. In this paper we present a study into using Virtual Reality in itinerant archaeological exhibitions. We discuss the lessons we have learned from developing an interactive Virtual Reality simulation of the Neolithic settlement of La Draga. These lessons feature our analysis of qualitative and quantitative feedback of museum visitors, as well as what we have learned from analysing their navigation and interaction patterns.

Keywords Virtual Heritage · VR analytics · Gamified experiences

\section{Introduction}

While it is important for archaeological museum curators to demonstrate excavated artefacts to let museum visitors witness those firsthand, it is probably even more important to educate the public about how these artefacts were used and to explain their significance in the cultural and technological development of the corresponding society. For many museum visitors this educational process happens through the mechanism of Informal Learning (IL), which is an instructional activity that is not part of the curriculum of educational institutions. IL provides the users with knowledge and understanding of a variety of subjects in museums, science centres, exhibitions, even in online communities or the workplace [1] [2]. The three types of Informal Learning, based on intention and awareness of the learning process, are: self-directed, incidental and tacit learning [3].

A cost effective form of Informal Learning is self-directed learning. Interactive Virtual Reality is a great technology that can effectively support this type of learning. In a virtual environment we can easily create static and interactive learning experiences that require little or no assistance from an educator.

One particular form of Informal Learning that we find useful for museum settings is blended learning [4]. Blended learning is often focused on combining online and face-to-face modalities, however, informal blended learning is generally conceived as "learning by knowing", i.e. passive learning through multimedia content facilitated by point and click or web-based interfaces [5]. Virtual Reality (VR) represents a modern alternative to point and click style interaction and is a useful technique for blended learning in a museum setting.

In recent years many VR experiences in museums have helped visitors to develop a coherent understanding of the corresponding society and the roles of the excavated artefacts featured in exhibitions [6] [7] [8]. For example, the Modigliani VR exhibition at the Tate Modern recreated the artist's studio [9] to let visitors take a virtual tour at their leisure. The British museum created a VR Bronze Age site [10], where users explored the landscape freely and could interact with objects while hearing the explanation. These and other 
experiences in museums have raised VR design issues such as modelling of 3D content, the strong connections between the VR experience and the physical artefacts in the museum, the lack of VR experience for many users [11], as well as making sure that the VR experience is sufficiently short (to avoid queues) yet educational. Nevertheless, there is still room for improvement such as designing more interactive experiences and making the user aware of their own informal learning.

In this paper we design and evaluate an informal blended learning experience in the context of cultural heritage dissemination, La Draga Neolithic settlement [12]. Our contribution is twofold: i) put together traditional methods in museums (in-site workshops conducted by expert archaeologists, and exhibitions of objects, videos and photos) with digital learning materials (a $360^{\circ}$ video with Samsung Gear glasses, and a gamified and interactive VR experience using HTC Vive headset); and ii) a study of user behaviour patterns when interacting in VR. We stress that the novelty of our research is the proposal of a hedonic, interactive and conscious learning experience, and the identification of user behaviour patterns using data analytics. Results show that visitors highly valued the blended learning experience, scoring it with 9,3 out of 10 . Moreover, they had a good perception of their learning, $85 \%$ of visitors considered they had learned during the exhibition. On the other hand, the study of user behaviours identified three profiles of users (Novice, Medium and Pro). Specifically, from the analysis of Newbies behaviour arised design recommendations useful for other researchers, such as the wise management of training tutorials, the incorporation of attractive and strategic locations to favour space exploration and the use of natural and adaptive interactions to avoid users struggling with VR devices.

In the following, section 2 presents related work. Next, section 3 introduces La Draga settlement and the process of construction of the Virtual Draga. Section 4 describes the design of the informal learning experience of La Draga. The design consists of a set of activities with the ultimate goal of delivering knowledge and understanding to the user. Section 5 presents results of the blended learning experience performed in a museum. Section 6 analyses user behaviour in the gamified VR experience, which was conducted in the museum as well as in several itinerant exhibitions. Section 7 discusses the results. Finally, section 8 concludes and presents future work.

\section{Related work}

There is no doubt that cultural heritage organisations may benefit from the use of digital technology to widespread knowledge and understanding of past cultures [13] [14] [15]. Applications for enhancing visitors' experience range from virtual guides in digital museums [16] to Augmented Reality (AR) [17] [18] and Virtual Reality (VR) [19] [20].

Focusing on VR, we find contributions from industry and academia. On the one hand, companies provide their services to cultural institutions, devel- 
oping historical recreations and virtual museums, such as, for instance, the virtual Dutch Men's EUseum [21] by ChroniclesVR [22]. On the other hand, several research works have studied the use of VR in museums during the last years [23], [24], [25]. Carrozino et al. [26] proposed a taxonomy of VR settings for cultural heritage applications and used it to analyse some case studies. The goal was to provide a tool for evaluating costs, risks, usability and quality of immersive experiences. Related to risks, they defined the "Guggenheim effect" as "the appeal of the container pushing into the background the essential element, the content, resulting a distracter rather than an opportunity to convey information". Our multidisciplinary research team, composed by experts on archaeology, 3D graphics, game design and programming, paid particular attention to disseminating the knowledge on Draga settlement (the content) while incorporating interactive and game elements in the experience (the container).

Gaitatzes [20] proposed a cave-based system to let museum visitors walk through various ancient sites, specifically, through the Greek city of Miletus. Encouraged by the high number of visitors and their positive feedback, they advocated for further development of cultural and educational experiences in museums. Another research used phone-based VR with the novelty of a homemade designed headset. This headset was a robust and safe hardware for long-term exhibitions, composed of an external battery, high quality headphones, and designed as a Viking wooden mask. Curators, technicians, artists, and archaeologists collaborated to design 3D vignettes of Viking settlements (scenes, objects and activities) in Ireland. They used fixed viewpoints, which constraint users' interactions with objects and their free movement around the exhibition space [24].

A recent VR research [25] studied the impact of different communication media (VR, website, video) on the UX (User eXperience) of visitors of the National Palace Museum in Taipei. Specifically, they presented the information about the Jade Cabbage to the users and measured their attention and engagement using questionnaires. They also aimed to study how users' personality and prior experience could influence the UX. Results highlighted the ability of VR to catch users' attention, and the influence of attention in increased engagement [20].

Previous works conceptualize and operationalize different edutainment VR experiences in museums and exhibitions. They were mainly designed containing virtual recreations (3D content depicting landscape, people and objects from the historic period) for the user to walk around, including virtual guides for visiting the space at most. Our research, with the aim of further engaging the user, incorporates the gamification element to target (hedonic-based) VR experiences. Moreover, we focus on the design of a game played by visitors that become aware of their learning during an exhibition, even encouraging them to eventually revisit parts of the exhibition after playing.

Related to blended learning in museums, the Blended Mobile Museum Learning Environment (BMMLE) was applied to three blended museum learning modes [27]: i) the traditional museum visit, ii) paper-based learning sheets 
used during museum visits, and iii) interactive mobile learning system used during museum visits, all of them accompanied by a learning website. We differ from them in the modalities of blended learning; while they studied online and mobile-based learning, we implemented experiential and active learning activities such as a workshop, and a VR experience. Additionally, as previously remarked, we included gamification and interactivity in the design of this VR learning experience.

Indeed, the interest in users' engagement led research works to use gamification in the context of museums. Thus far, however, these works were limited to contributions on design and methodological perspectives, neither implemented nor evaluated. In [28] several use cases of a gamified museum guide were designed and evaluated. Although authors revealed some weaknesses of the evaluation method, which was strongly based on subjective criteria, they bet for meaningful gamification based on a coherent story and with a limited number of game design elements. Another work [29] presented a framework of user experience for museum based on gamification. They considered important to understand museum audiences and so meet the needs and expectations of users' profiles. In our research we take a different perspective to previous works that focused on theoretical contributions. We aim to go through the entire process from the design, to the deployment and the evaluation of gamification in a museum.

Finally, regarding adaptive technologies, museums around the world adopted them to customise information for different target groups in web applications and hand-held devices [30] [31]. Another research study [32] used observation and questionnaires answered by visitors of museums, with the aim of studying their visiting style. Styles were defined based on animals: an ant visitor follows a linear and clear path, a fish visitor sees the big picture of the exhibition and does not stop frequently, a butterfly visitor moves non-linearly, and does not pay attention to the curators' indications, and finally, a grasshopper visitor has particular interests and only approaches certain items, spending significant amount of time in front of them. They found a high correlation between visiting styles and cognitive styles. Using the knowledge gained by them, and making an analogy between the visit to a museum and the visit to a virtual recreation of a historical settlement, we designed our experience as an open-world with free movement, trying to cover the variety of aforementioned visiting styles.

To our knowledge there are no other works based on data analytics to study user profiling in Virtual Reality heritage projects. However, in recent years profiling in games has gained attention for informing game design, ROI, study of human behaviour, and driving adaptive systems [33]. Multi-game social networking platforms like Steam provide analysts with data from million of players. Unlike traditional profiling methods based on surveys and observations (as proposed by Bartle [34]), nowadays a myriad of AI (Artificial Intelligence) techniques (k-means, decision trees, random forest) are used to build players profiles [35] [36]. In this research we perform a preliminary study of 
user profiling for VR in museums. We use aggregate statistics and basic ML (Machine Learning) clustering.

\section{La Draga settlement: Reconstruction of the past}

La Draga settlement is an Early Neolithic pile-dwelling settlement located at the NE of the Iberian Peninsula, on the eastern shore of the Banyoles Lake, dated back 5.300-4.800 BC (see first row of Figure 2). Its singular location, partially under water, has facilitated a high degree of conservation of its archaeological remains, highlighting the preservation of items elaborated with wooden and vegetal fibres - an organic material rarely preserved. This fact allowed the study of technical procedures used to manufacture such tools and some construction elements. Moreover, archaeologists have hypothesised and reconstructed the buildings' architecture, as well as achieved knowledge about the subsistence and the working activities performed by the Neolithic farming society in that environment. All the reconstructed data comes from previous palynological $^{1}$, carpological ${ }^{2}$ and anthracological ${ }^{3}$ studies [37] [38] [39] [40]. Additionally, although no information exists about the appearance of Draga's inhabitants, and neither about their customs, data about people and animals came from ethnographic resources, from Levantino rock-art paintings and archaeological investigations elaborated at other sites from the period.

However, despite the importance of the site due to its well-preserved remains, there is still room for promoting actions to attract more visitors and to disseminate La Draga beyond the Banyoles settlement.

\section{Informal learning design of La Draga settlement}

The main goal of the archaeological community was to design an itinerant exhibition to spread the spirit of La Draga to the general public. Moreover, they wanted to provide a holistic experience to visitors so that they not only could feel and understand the settlement, but also they would be aware that they had retained new knowledge at the end of the experience, arousing visitor's curiosity to go to the physical site. Specifically they were interested in the visitors experiencing La Draga, the way its inhabitants lived, what they ate, the activities that they carried out beyond agriculture and livestock, etc.

To obtain such a holistic experience, a blended learning approach seemed to be appropriate. It combines educational materials, experimental workshops and virtual experiences in a whole exhibition. We decided to include noninteractive and interactive activities letting visitors explore and experiment

\footnotetext{
1 Palynology is the science that studies live and fossil spores, pollen grains, and other microscopic plant structures.

2 Carpology is the study of the structure of seeds and fruit.

3 Anthracology is the analysis and identification of charcoal which is preserved after carbonization, based on wood anatomy.
} 
respectively, as "experts" of the past. First, we designed physical activities, where attendants could watch preserved pieces of La Draga, could explore a physical reconstruction of a hut, could watch short films concerning the activities of the daily life of Neolithic inhabitants, and could experiment the technique for carving flint stones. Nevertheless, all these activities in an itinerant exhibition could not reflect the whole environment neither help visitors place objects in context. Second, the incorporation of VR appeared as a powerful way to enable visitors to contextualize objects and activities as well as to provide them with a digital experience in line with the digital era we live. We designed two different experiences to reach different types of users: those reluctant to VR technology - a 360-degree walk becomes an easy introduction to VR -, and bold users eager to face new challenges - for which interactive $\mathrm{VR}$ is more appropriate.

Our first digital experience was a 360-degree video of a reconstructed 3D virtual scene that guides users through the environment of La Draga, a set of objects, huts and activities at their own pace. The second one was a VR immersive experience where users could freely explore outdoors, manipulate objects and activate animations. Moreover, in this second approach, to make visitors aware of their learning during the exhibition, we included a little game with the main goal of distinguishing non-Neolithic from Neolithic objects.

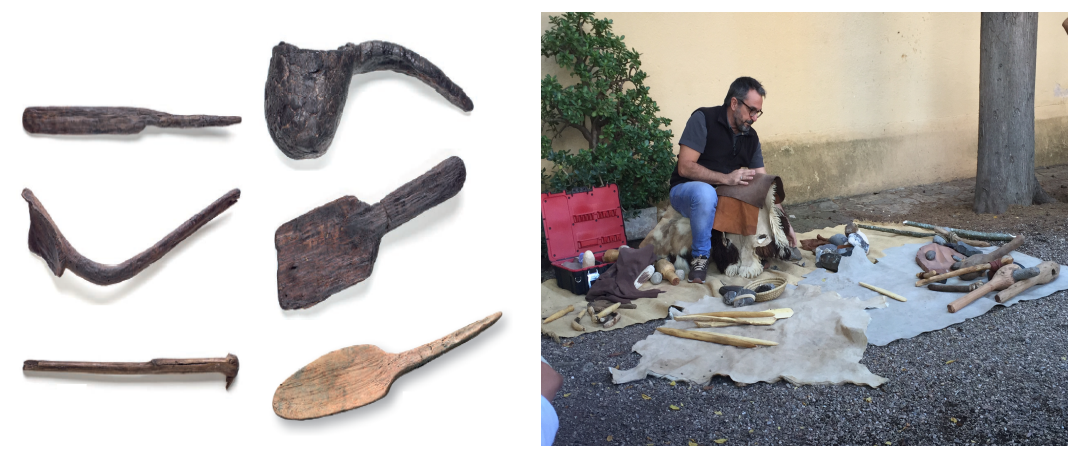

Fig. 1 Some representative objects of La Draga and flint knapping workshop.

In summary, we conceived six different resources to be located in a shared space: (1) an exhibition of two hundred archaeological heritage preserved pieces of La Draga settlement, (2) a physical reconstruction of a decorated hut, as they have appeared in the excavation and that can be manipulated, (3) eight videos that show the peculiarities of the archaeological team working around the settlement and bring the vision of leading specialists on several topics (Ferran Adrià - cooking and food, Martí Boada - human footprint on the environment, Marc Boada - technological innovation, and Eulàlia Subirà the origins of the community Draga from the paleogenetics), (4) experimental workshops to learn the flint knapping (see Figure 1), (5) a $360^{\circ}$ virtual reality 

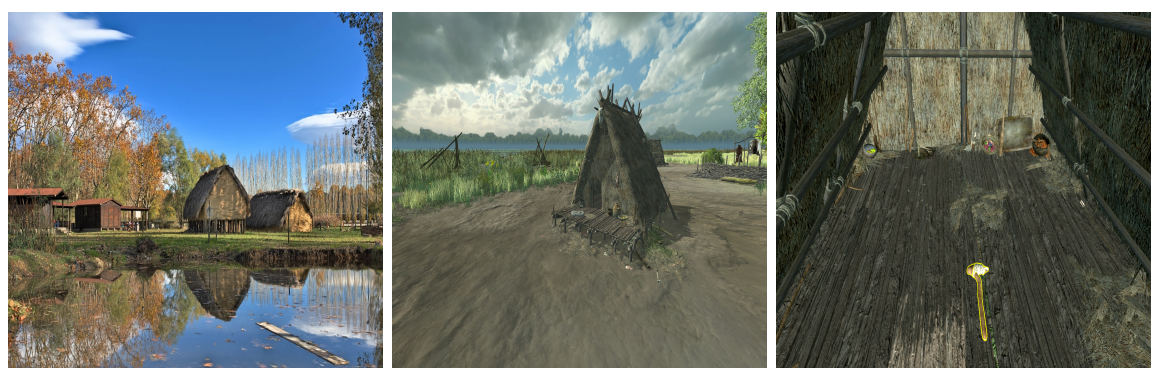

Fig. 2 On the left, La Draga settlement on the shore of the Banyoles Lake. In the middle, a perspective of the virtual reconstruction, and on the right, the interior of a virtual hut.

walk through the site and its surroundings, and (6) an interactive VR game to explore the objects and activities.

\section{VR $3 D$ modelling decisions}

The last two proposed activities required the creation of $3 \mathrm{D}$ virtual scenes containing the 3D environment, 3D objects as reconstructions of their counterparts found by archaeologists, and animations of inhabitants performing daily life actions. The design decisions related to these $3 \mathrm{D}$ scenes have been reached by mutual agreement between archaeologists, arts designers and computer scientists involved in the team. All VR elements and actions have been designed based on these archaeological and archaeometrical studies, as well as on laboratory experiments carried out (see Figure 2, in the middle): the landscape, the land usage, the architecture of buildings, the working activities and the tools used to develop these activities. We opted for realistic instead of photorealistic rendering to convey users a possible re-created reality of the past based on present-day knowledge, though that reality might not exactly match the existing one.

Specifically, for the 3D reconstruction of La Draga the landscape and its usage, three small wheat fields were reconstructed. One is presented already harvested while the other two simulate part of the village. All of them located on the shores of the Lake Banyoles, in a similar area where the settlement is currently located. This large composition lets users have an extensive and complete view of Neolithic environment.

In order to model the huts as reliably as possible, we have based ourselves on the results of morphometric and spatial analysis of the wooden architectural remains in the settlement [41], and on the recent results on the 3D reconstruction and modelling of ancient houses [42]. As huts are one of the most important archaeological results of La Draga, we decided to model them accurately, with a lot of internal and external details, to let users to enter, to explore their innermost, and feel what it was like living in such a home.

Related to the 200 objects exhibited in the museum, we chose to include 4 relevant objects (a bow, a sickle, a ladle and an adze) in their likely places in the virtual Draga. We selected these key objects since they were well-preserved pieces in the physical settlement, and they were also significant tools in the 
daily life in La Draga. We also decided to include some animals and fruits of the epoch (a deer, a pig, an apple) and three inhabitants doing daily activities with some of the included tools. These three activities replicated some parts of the flint knapping workshop and were modelled as predefined animations. As we mentioned before, no data exists about Draga's inhabitants and animals. Then, to create the VR characters, we collected the data from near sites of the same period. It has to be highlighted that the majority of the ornamental stuff applied to the characters are evidences found at the settlement [43].

A professional illustrator modelled the 3D scenes with textures and animations using Blender ${ }^{4}$, always collaborating with specialists of each study area to preserve the maximum reliability of the VR data. All the assets were included in two Unity ${ }^{5}$ projects with their corresponding scripts and shaders, using a desktop setup to test all the functionalities before VR deployment.

All the aforementioned decisions were made for modelling the 3D space used as a basis for the two VR experiences. In the next section we will detail both of them.

\section{Design of VR experiences}

On the one hand, the $\mathbf{3 6 0}^{\circ}$ virtual reality walk through the site and its surroundings was designed following the observations of archaeologists. Fields were located outside the settlement, in a highly deforested oak forest with numerous clearances. This virtual tour through the fields allows users to watch 5 -minute-long cinematic reconstruction of the settlement. We considered that this duration was enough because we could better capture the visitor's attention as they would only see the best parts of La Draga, leaving them enough time for the rest of activities. We designed the virtual walk as a peaceful stroll including a surprise as a way of entertainment. For example, as the walk continues, a deer would suddenly appear from the bushes (see the full video at https://youtu.be/ojbw6IuXIVQ).

On the other hand, the design of the interactive and gamified VR experience aims to engage and make participants aware of their learning about objects of La Draga. Visitors play the role of a time traveller. They have 5 minutes to get rid of all the Non-Neolithic objects that they encounter and have to throw them to a time portal. We established a time constraint of 5 minutes, which is recommended in a museum setting [27] as too many people could be queuing and waiting during exhibitions.

In addition to the Neolithic objects, food and animals referred to previously, we modelled 6 non-Neolithic objects (a saw, a modern Bow, a Knife, a Dart, a banana and a Cola Can). We selected them to help visitors realise if they had learned that iron-made tools belong to the non-Neolithic era and stone and wooden tools to the Neolithic. We did the same with fruits, banana and apple as non-Neolithic and Neolithic respectively. We also included a Cola Can as an obvious challenge to facilitate game on-boarding.

\footnotetext{
${ }^{4}$ Blender is a 3D modelling and rendering package, http://www.blender.org

5 Unity Engine is a Game Engine Platform, http://docs .unity3d.com
} 
The game scene was structured in different zones (see Figure 3). In each game several objects, which may be repeated, are randomly placed in certain locations of the site (coloured circles in Figure 3). This way, each game is a little different from the previous one. When players throw a non-Neolithic object into the portal, they score 100 points and lose 50 for each mistake (i.e. when they throw a Neolithic item). The player lands at the point marked as a blue diamond, near to the time portal which is depicted as a yellow star (see "Landing and Portal" zone in Figure 3).

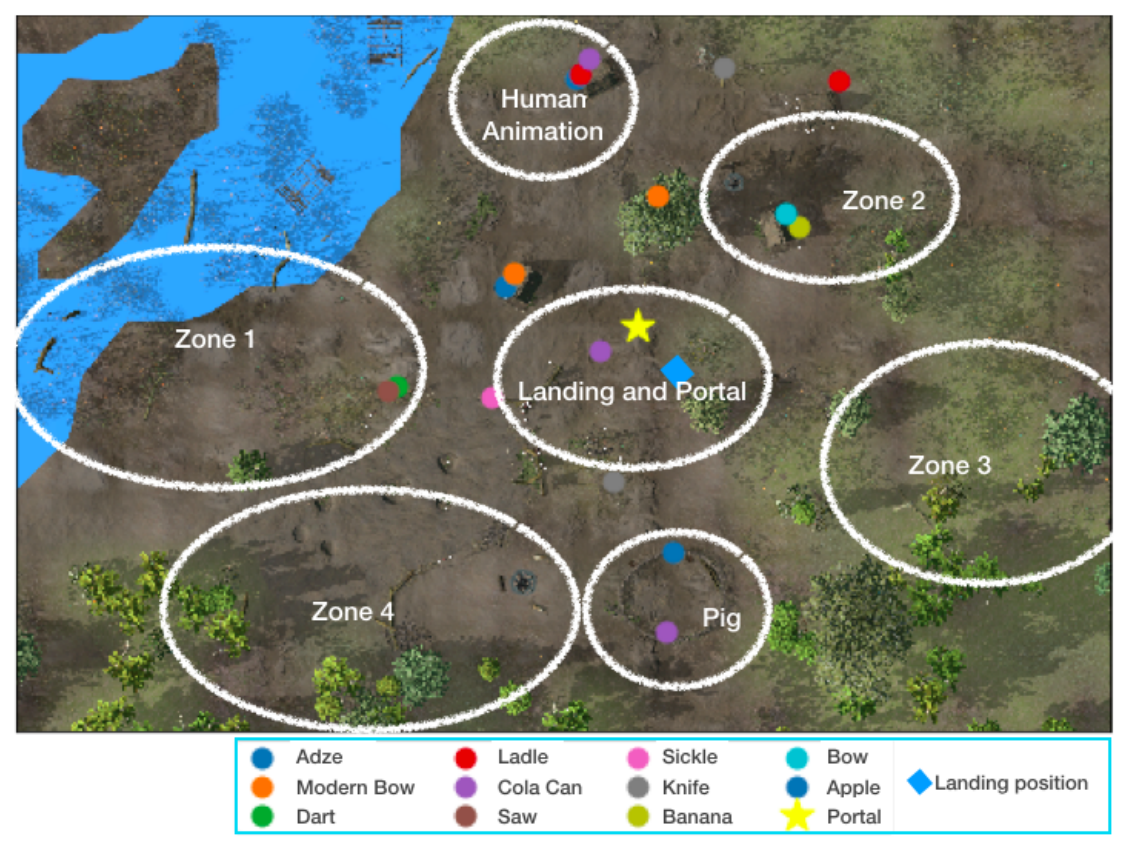

Fig. 3 Objects distribution. Blue diamond depicts the landing position, the yellow star depicts the time portal, and the coloured circles are objects. The Neolithic objects are: a Bow, a Sickle, a Ladle, an Adze and Apples as pig's food. The Non-Neolithic objects are: a Saw, A Cola Can, Bananas, a modern Bow, a Knife, and Darts.

Additionally, to reinforce the fun, we included some quests and surprises throughout the game. First, we included a wild pig that guards a "Cola Can" (a Non-Neolithic object). The player must distract the pig by throwing apples, while the pig leaves his area, the player can pick up the Cola Can (see left part of Figure 4). If the wild pig comes back too soon, it attacks the player by surprise. Second, we added an interaction with a Neolithic inhabitant animation when the player grabs a Neolithic sickle located in a hut of the site. This animation shows a flintknapper, reconstructing the experimental workshop offered in the museum (see the right part of Figure 4).

Other decisions made when designing the VR experience were related to the VR interaction mechanisms. We included teleporting to support player 

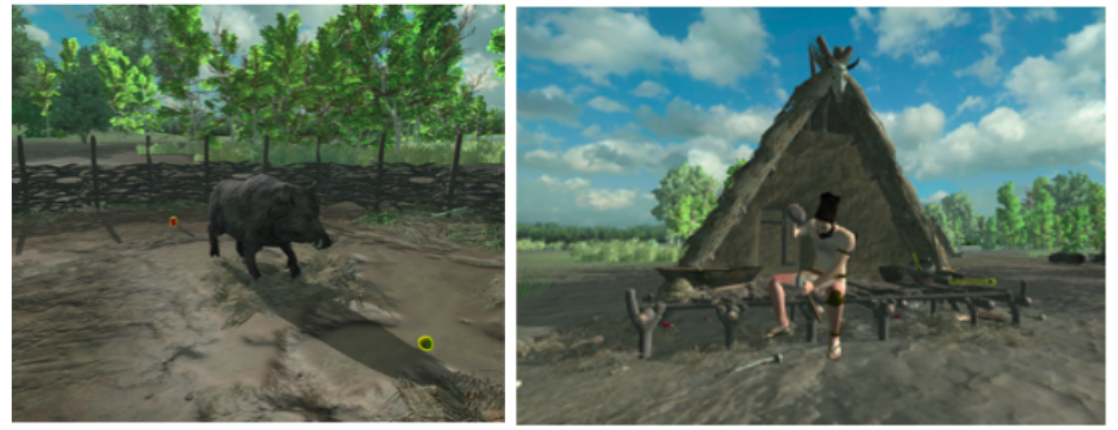

Fig. 4 Pig and Human.

movement through the site, grabbing objects to collect items, and throwing objects into the time portal. The trigger buttons of the hand-controller are used for teleporting, depicted as a parabola, allowing users to control the end point of the movement (see right part of Figure 5). Additionally, we highlighted the selectable objects with a yellow border so that the user can easily detect and grab. Users must pull the trigger control to pick up and grab the selectable objects (watch the VR defined interaction shown in the video https://www . youtube. $\mathrm{com} /$ watch? $\mathrm{v}=\mathrm{xmAe} 0 \mathrm{zx} 8 \mathrm{KrU})$.

\section{Technical Setup}

Since we planned itinerant exhibitions with short-term VR deployment, we required technical staff to move the exhibition between museums. Staff technicians were tasked with configuring the VR setting, with helping users to appropriately wear VR devices, and with solving problems that might occur during exhibitions. We employed 4 Samsung Gear VR glasses with headphones (for the 360-degree experience), and one HTC VIVE headset with wired HMD (Head Mounted Display) and wireless hand-controllers (for the VR game). We opted for that number of Gear VR glasses because of their affordable price and their easy configuration. Easy manipulation was particularly important for facilitating their use by museum staff. We only employed one HTC VIVE headset due to the limited number of technical staff that we had on the site. As a requirement of the HTC VIVE room-scale VR, museum managers were asked to enable a large zone (at least $3 \mathrm{~m} \mathrm{x} 4 \mathrm{~m}$ ) in the exhibition area. This location helped users revisit the physically exhibited materials (objects, panels, videos) after their VR experience, thus they could reinforce their learning. Furthermore, we showed the VR games as played by users on a large TV display to share the VR experience with the users queuing to play.

The following two sections present the results of two studies that evaluated activities organised around La Draga. The first study focused on the assessment of the blended learning experience in one of the museums of the itinerant exhibition, and the second one aimed at analysing user behaviours during the VR experience. 


\section{Evaluation of the informal learning experience}

The goal of the evaluation was to obtain a first overall impression from users regarding: perceived learning in the museum, how physical and virtual experiences complement each other, and environment realism.

\subsection{Methodology}

We recruited 42 voluntary users that visited the "Museu d'Arqueologia de Catalunya" during two consecutive weekends. The users that participated in the study include families with children, usual museum visitors and tourists. According to sociodemographics studies about early VR adopters [44], we expected users to have a variety of skills on interactive applications such as games and VR, ranging from kids that are used to play games frequently, and occasionally VR, to those that have neither gaming nor VR experience.

As museum visitors arrived, they were informed about La Draga exhibition, and the activities organised around it: workshop conducted by an expert archaeologist in Neolithic, Draga in $360^{\circ}$, and the gamified VR experience. Most of the visitors went to the workshop, visited the museum, and took part in at least one of the two virtual experiences (either $360^{\circ}$ video-walk or the VR game).
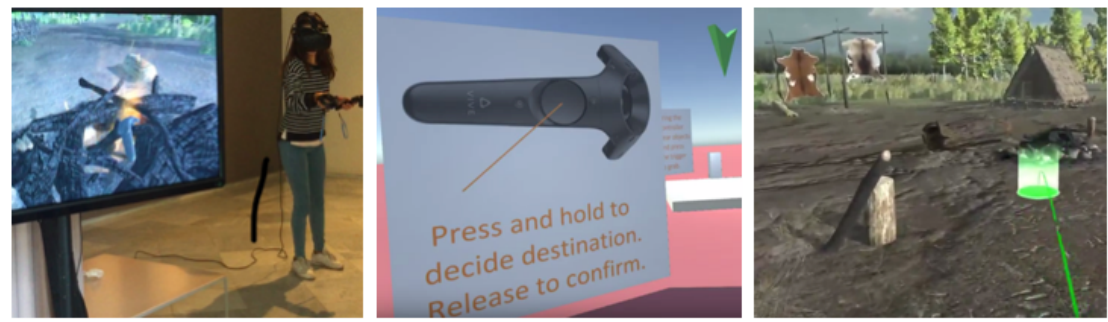

Fig. 5 On the left, VR experience at the Museum. On the right, some captures of the on-boarding tutorial and the game in action.

Those visitors that participated in the gamified VR experience filled a postexperience questionnaire and were informed about the anonymity of the data gathered (see Figure 5).

This questionnaire helped us to gather users' perceptions, attitudes, and opinions. It contained closed-ended and open-ended questions that aimed to collect information about the two main aspects of the experience: a) perceived learning and value of blended learning; b) and aesthetics and engagement (see Table 1). Questions Q1 to Q6 had as possible responses: A lot, Enough, Somewhat, and Almost nothing. Question Q7 had a numerical scale from 0 to 10 . 
Table 1 Closed-ended questions of the post-experience questionnaire.

\begin{tabular}{|l|l|}
\hline \hline Informal blended learning experience & \\
\hline Q1 & $\begin{array}{l}\text { Is the VR experience a good } \\
\text { complement to the exhibition? }\end{array}$ \\
\hline Q2 & Have you learned in the experience? \\
\hline Q3 & $\begin{array}{l}\text { Have you recognised exhibition's } \\
\text { objects in the VR experience? }\end{array}$ \\
\hline Q4 & $\begin{array}{l}\text { After the VR experience, have you built } \\
\text { up an idea about La Draga } \\
\text { settlement? }\end{array}$ \\
\hline VR added value: realism and engagement & \begin{tabular}{l} 
Have you enjoyed the VR experience? \\
\hline Q5
\end{tabular} \\
\hline Q6 & $\begin{array}{l}\text { Was the design of the virtual Draga } \\
\text { realistic? }\end{array}$ \\
\hline Q7 & $\begin{array}{l}\text { Rate the VR Draga experience (from } 0 \\
\text { to 10) }\end{array}$ \\
\hline
\end{tabular}

\subsection{Results}

In the following we analyse data gathered from questionnaires as shown in Figure 6 .

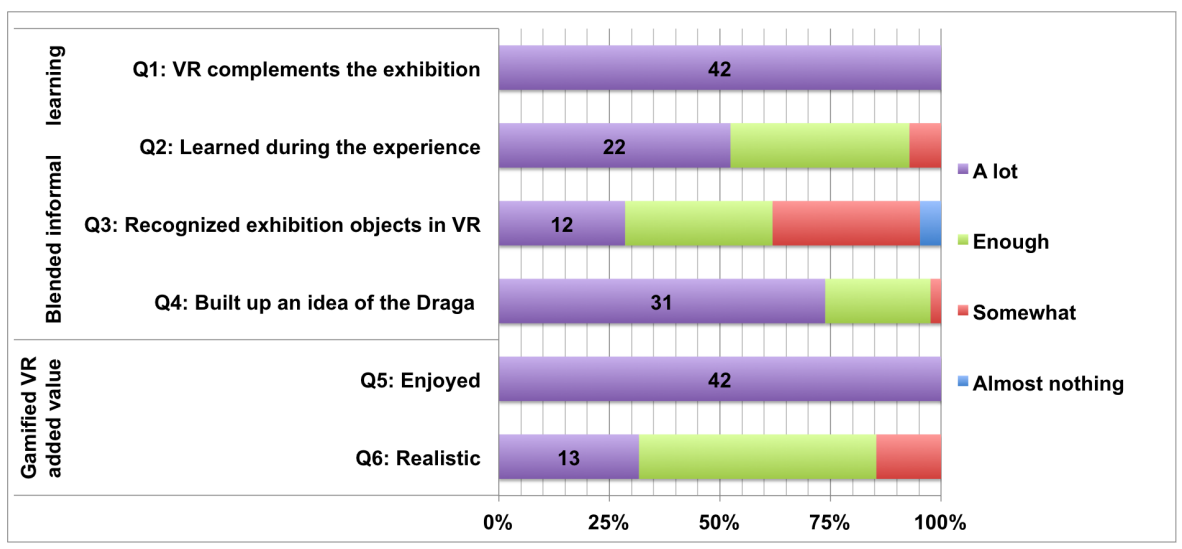

Fig. 6 Quantitative analysis of closed-ended questions Q1-Q6.

Regarding the informal blended learning experience, results are quite encouraging. A particular highlight is Q1, the question related to considering the VR experience as a good complement to the exhibition, where $(100 \%$ of users gave the most positive answer to Q1). Moreover, perceived learning in the VR experience also gives good, but lower, scores (Q2). This is probably because of the experience duration. It was short (with an average of no more than one hour) between workshop and museum visit (45 minutes), $360^{\circ}$ (5 minutes), and the VR session (5 minutes). 
The results of Q3 - "Have you recognised exhibition's objects in the VR experience?" - show that more than half of the users found a significant number of exhibition's objects during the gamified VR experience. We think that the rest of users did not recognise many objects because they spent a considerable amount of the 5 minutes struggling with interaction techniques (teleporting, grabbing, throwing), thereby preventing them to further explore the virtual space in the search of objects.

Regarding Q4 - "After VR experience, have you built up a good idea about the La Draga settlement?" - almost all users perceived that the VR experience let them enhance their knowledge about La Draga.

Questions addressing engagement and aesthetics (Q5 and Q6, respectively) yielded mixed results. On the one hand, the enjoyment of the experience reached the most positive opinion from all users (surely due to the novelty of the immersive experience, and the proposed game). On the other hand, although we put a lot of effort into the modelling a trustworthy Draga reconstruction, only $30 \%$ of the participants thought it was very realistic in comparison to $50 \%$ who thought that it was correct enough. There could be multiple reasons for such responses. First of all, the phrasing of the question may have implied that "realistic" was to do with photo-realism rather than historical accuracy of the visualisation. In addition to that, users were unaware how historically accurate the exhibition was, so they had to guess. Another possible reason is that before to the workshop and the exhibition users saw real items and pictures and might have found virtual virtual objects being less realistic than the real excavated artefacts.

Some visitors preferred to find real objects instead of their virtual counterparts, whereas others expected to encounter inhabitants in the virtual Draga. Nevertheless, we think that the problem may not lie entirely with the design of La Draga, realistic enough to allow real time interactions, but with users having high expectations triggered by a photo-realistic renderings that they saw throughout the exhibition.

As for the remaining questions, 97\% of users scored La Draga VR experience (Q7) at 8 or above (in a scale of 10). Only one user rated the experience with 6 points. However, as it is evident from the user's comments, this user struggled with the VR controller. This fact is also confirmed by our analysis of the corresponding interaction logs.

Additionally, using non-parametric statistics, we found a moderate significant correlation (Spearman $\rho=0,340 \mathrm{p}=0.05$ ) between scores of perceived learning (Q2) and those considering the 3D reconstruction useful for better understanding La Draga (Q4). It confirms that the VR Draga actually contributes the informal blended experience. On the other hand, there was no correlation between (Q4) and the perceived realism (Q6). The reasons for this could be question wording and together with other issues we mentioned earlier.

We also conducted qualitative analysis of open-ended questions. Responses were analysed and, if appropriate, grouped according to categories. As the result of this exercise we received an insight into visitors' opinions and feelings in relation to the virtual Draga. 
When asked "How the blended learning experience has helped you to learn about La Draga?", users provided two types of answers, one directly related to the blended learning experience, and the other related to the scenography. One subject wrote "I was in the place that I had just seen in the exhibition, I was in La Draga!". Most of the users refereed the scenography. Also users showed good understanding of La Draga in relation to village size and layout, surroundings and landscape, cabins, and even animals (i.e pig). A number of visitors learned that there were apples in the Neolithic. Indeed, they used the apples to feed the pig that was inside a fence.

Another question was "Which details have you found in the immersive experience that you did not notice in the museum?" Respondents mentioned the realistic environment, the interaction, and being immersed in La Draga as a Neolithic inhabitant. Therefore, we see that users, as in the previous question, highly valued the experience of being in La Draga, and also appreciate the interactive nature of the experience.

Responses to the question "What would you change in the gamified VR experience? Please provide details that have pleased you in the exhibition?" were rather interesting. We group them into two categories: the design of the gamified experience, and the design of La Draga environment. Firstly, a number of users asked for a longer experience because they needed more time to learn how to use the VR controllers. This again reinforces our hypothesis that the VR interaction problems had a significant impact on the user experience.

Finally, regarding the question "Do you think a virtual reality visit would be better than a physical visit to the exhibition? Why?". We arrange gathered opinions in three categories: i) virtual and physical visit complement each other, ii) VR is more comfortable and useful if you can not go to the museum, and iii) just to visit the exhibition in the museum is enough, because you can see things in reality. Most responses fit into the first category (as also showed in close-ended question Q1), and few in the second and third categories.

In summary, quantitative and qualitative analysis of questionnaires showed that users were really satisfied with the experience. They put the focus on the goodness of the informal blended learning, i.e. complementarity of physical and virtual activities. And, despite of difficulties with VR interaction faced by some users, almost all of them rated the virtual experience very high. Indeed, Norman [46] states that users are prepared to put up with certain negative aspects of an experience if they find it appealing and aesthetic.

\section{VR interaction evaluation}

The goal of the evaluation was to study gameplay and VR logs to get insights into users' behaviours. To do so, we logged users' interactions during the entire experience, analysed the data, and aimed to infer behaviour patterns useful for improving the design of cultural-heritage VR experiences. 


\subsection{Methodology}

We recruited 262 voluntary users with diverse backgrounds that visited the museum of archaeology and attended different itinerant exhibitions. User profiles included academic practitioners, archaeology experts and general public. All participants were equally distributed in terms of gender. Regarding the age, $50 \%$ of users were between 22-35 and the rest of them were between 36-65 years of age. The majority of users had medium-to-low experience in games and VR applications.

Users that approached our exhibitions were invited to participate in La Draga VR experience, and were informed about our research purposes and the anonymity of data collected. In our game, user had to move around the virtual Draga, find objects and throw them to the portal only if they did not belong to Neolithic era. Therefore, the logs provided data of main VR interactions related to game score, navigation and interaction with objects.

We collected the data needed to evaluate the interaction behaviour, spatial behaviour and performance behaviour (regarding the game goal) of each player. Thus, during each game play, we continuously tracked the location of the player and monitored their interactions in terms of teleport actions, grabbing (and regrabbing) of objects, throwing of objects at the portal, and orientation changes by head rotation. We found that such information was enough to characterise the aforementioned user behaviours. Then, logs were structured with the following elements (attributes in parenthesis):

Timestamp, Player (position, orientation) Teleport_From_To (positionF, positionT), Grabbed_object (idObject, posObject), Score (corrects, mistakes), Pig_Interaction, Human_Anim.

First four elements are self-explanatory and the last two indicate whether the player approached the areas of the pig and the virtual human, and interacted with them (see Figure 4). On one hand, pig interaction consisted of throwing an apple to distract it and grabbing an object situated near the animal. On the other hand, the interaction with the human started an animation of a Neolithic inhabitant.

\subsection{Results}

We used unsupervised classification for player behaviour analysis [47]. More specifically, the k-means method with parameter $\mathrm{k}$ equal to 3 . Figure 7 shows the result of the clustering at the end of the game. It was performed with the features: total number of teleports, grabbing efficiency, and game score. Note that grabbing efficiency is computed as the number of successful grabs divided by the total number of grabs. Unsuccessful grabs represent the re-grabbing of objects that users threw to the portal, did not enter and had to grab again, as well as objects that fell from their hands.

The three coloured areas (red, green, purple) depict three different behaviours ("Novice", "Medium", "Pro") of users that played the virtual Draga. 


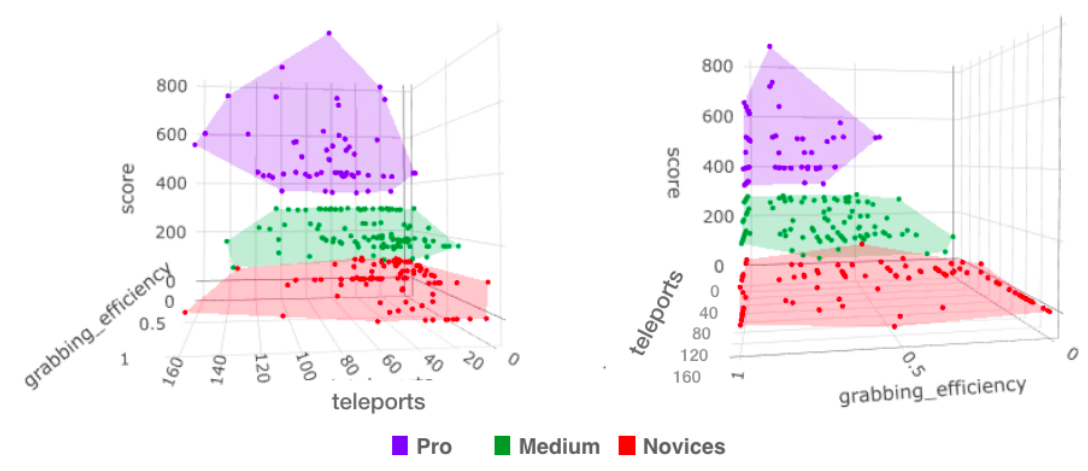

\begin{tabular}{l|ccc|ccc|ccc}
\multicolumn{4}{c}{ grabbing_efficiency } & \multicolumn{3}{c}{ teleports } & \multicolumn{3}{c}{ score } \\
\hline Cluster & Mean & SD & Median & Mean & SD & Median & Mean & SD & Median \\
\hline Novices (94) & 0.50 & 0.36 & 0.50 & 34.7 & 27.0 & 28.5 & 52.6 & 49.9 & 100.0 \\
\hline Medium(112) & 0.75 & 0.19 & 0.73 & 56.8 & 26.8 & 51.0 & 233.0 & 50.0 & 200.0 \\
\hline Pro(56) & 0.86 & 0.14 & 0.88 & 84.7 & 25.6 & 80.5 & 457.1 & 95.0 & 400.0 \\
\hline
\end{tabular}

Fig. 7 Clustering at the end of the game. In parenthesis, cluster size. Total number of users: 262 .

Red cluster (35,8\% of users) represents novice players, who scored very few points and rarely teleported. Regarding objects manipulation, grab efficiency varies significantly, lying in the interval $[0,1]$. The green cluster depicts the behaviour of Medium players (42,7\% of users) that achieved medium score. Users in this cluster performed a low-to-medium number of teleports and their grab efficiency situates in the interval $[0.25,1]$. Finally, Pro players (21,3\% of users) are in the purple cluster. They have the highest scores, a medium number of teleports, and grabbing efficiency is in a (higher) tight range [0.5,1].

The Table in Figure 7 shows statistics of features (grabbing efficiency, teleports and score) of these clusters. It should be noted that the mean of all the features increase from Novices to Pro users. The only statistic that remains stable between clusters is the Standard Deviation of teleports. Actually, it means that all the clusters contain some "explorers", i.e. users who needed a large number of teleports to navigate through the settlement.

Figure 8 shows the position of the Novices, Medium and Pro users of Figure 7 at the half time of the game. While Medium and Pro users moved up the leader board towards the end of the game, due to their increased their score, most Novices have not improved their ranking.

Moreover, to analyse the evolution of the user performance, we also clustered users at the half time of the game (shown in Figure 9). We detected some migrations between clusters. Some users improved their skills $(11,60 \%$ of final Medium players came from Novices at the half time of the game, and $21,42 \%$ of Pros was Medium before), whereas others became noticeably worst 


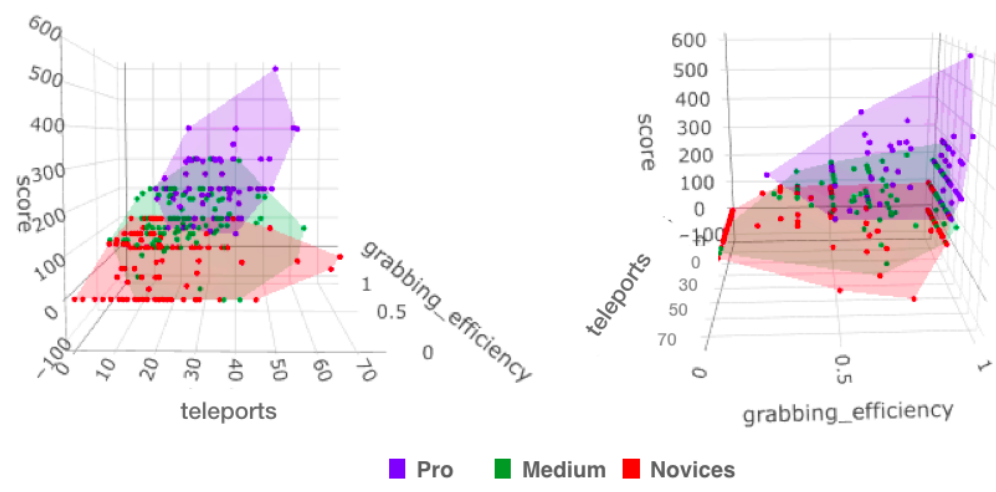

Fig. 8 State of the final clusters at half of the game in relation to the grabbing efficiency and the total number of teleports.

(30,35\% of Medium players were Pro at the half time of the game, and 32,97\% of final Novices came from the Medium cluster).
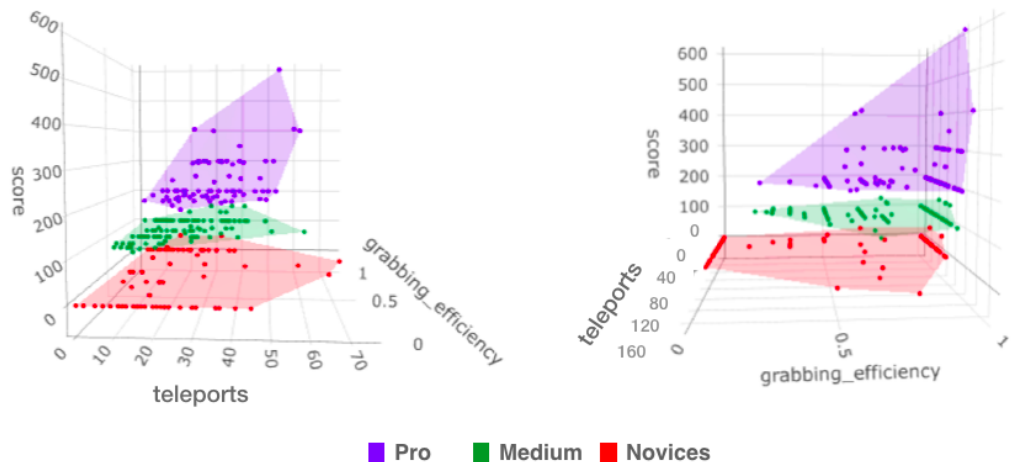

Fig. 9 Clusters at the half time of the game in relation to the grabbing efficiency and the total number of teleports.

We are especially concerned with Novice players at the end of the game (those that became worst during the game, and those that were Novices since the beginning). To better understand users in this cluster, we traced back their paths while visiting the virtual Draga. We found four patterns based on variables like Visited Zones, Score, Number of Teleports, Grabs, Failed Throws, and Pig and Human Interactions. Figure 10 shows the four patterns: "Struggler", "Engaged", "Explorer", and "Late-Explorer".

Strugglers are those Novice users that really had problems while interacting in VR to move and interact with objects (23,6\% of Novices were Strugglers). 

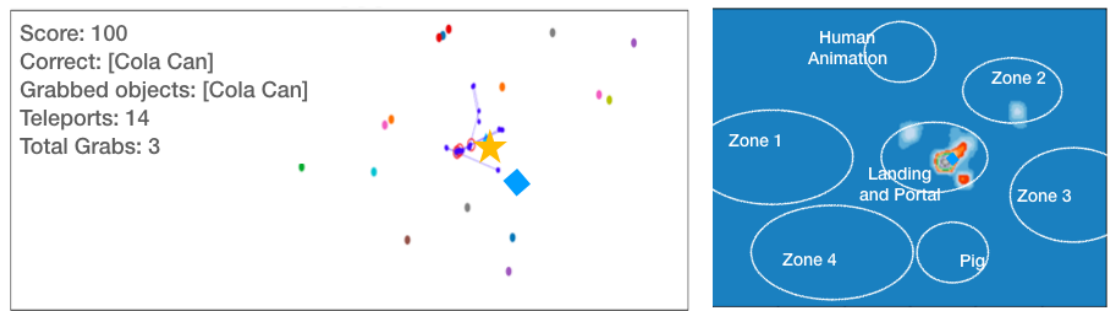

(a)
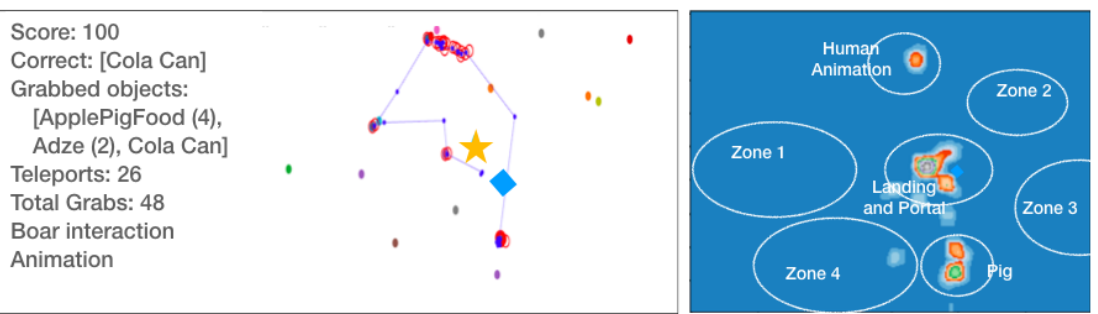

(b)
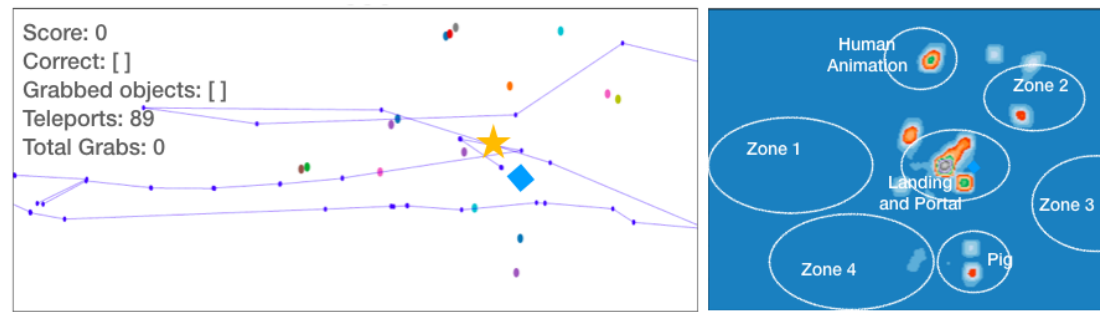

(c)
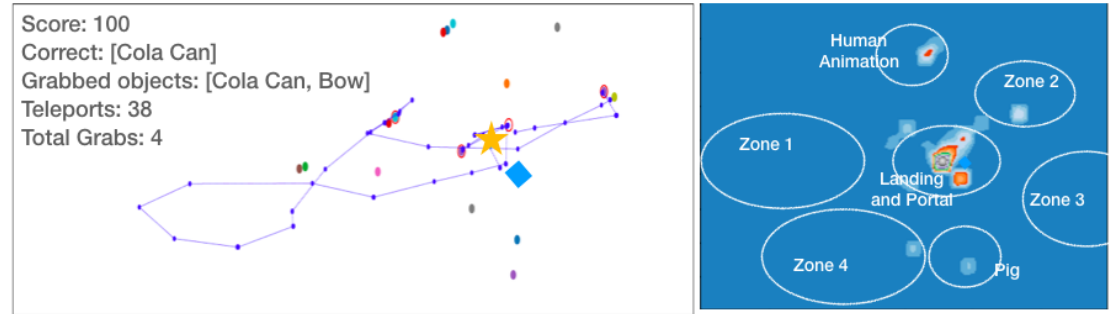

(d)

Fig. 10 Paths of the different behaviours : (a) Struggler, (b) Engaged, (c) Explorer, and (d) Late-Explorer. A filled red circle means that the user grabbed an object from its initial position (as was situated in the initial distribution), and empty red circles denote the user was regrabbing an object that was previously thrown from its initial position to the portal, and probably the user did not succeed putting the object into the time portal. On the right, heatmaps depict the corresponding behaviours.

Therefore, they visited few zones in the virtual Draga, just the landing area 
near to the 'time portal' and the vicinity. Figure 10 (a) shows on the right a representative example of Struggler behavior. Coloured dots represent Neolithic and non-Neolithic objects, blue dots and red circles depict teleports grabbing gestures respectively. On the left, the heatmap of all Strugglers shows that they just visited the Landing and Portal zone.

The rest of users in the cluster of Novices did not achieve a good score either because spent too much time with the pig and the animated human, or because they decided to explore instead of playing. In the following, we describe each of these cases.

Some Novices were not actually Strugglers because they preferred to play and interact with the game, namely Engaged users. They spent time in the pig challenge or interacting with the animated human (13,1\% of Novices were Engaged). Therefore, their paths show few visited zones. Consequently, they gathered few objects and received low game scores, however, they reported of having had a good time. Figure 10 (b) displays the behaviour of a typical Engaged user, who visited the fence and feed the animal with an apple (the fruit acted as a distraction in order to grab the Cola can that was situated below the pig). The animal charged at the player provoking a sudden fear and posterior loud laugh.

The most typical behaviour of Novice players is the one that defines them as Explorers (50\%). That is, many users visited several zones of the virtual Draga (see Figure 10 (c)). Indeed, a low number of grabs (in the example of the Figure this number is 0) denotes that they were not interested in the game but were keen to walk around and learn about La Draga.

Finally, Late-Explorers (see Figure 10 (d)) represent those visitors who had interaction problems with VR controllers at the beginning of the experience $(10 \%)$. That is, near the landing position. These users had some grabs and failed when throwing objects at the portal. Then, probably frustrated, they decided not to play but to have a walk and explore La Draga.

\section{Discussion}

In this section, we go through findings and analyse them. Moreover, building on lessons learned we provide design recommendations useful for other researchers who face the design of blended learning experiences including VR games.

\section{Blended learning}

The results of the first evaluation study show that visitors highly valued the blended learning experience. Definitely, the multi-modality of learning materials - favoured by physical and digital activities (photos, videos, live demos, interactive VR game) - helped museum visitors to learn about La Draga [48].

Indeed, the high scores forthcoming from questionnaires show that users i) enjoyed the gamified VR experience and perceived that they had learned about the Neolithic era, and ii) liked to identify 3D virtual objects, landscape and places, previously seen in the museum exposition, denoting that the virtual 
reconstruction of La Draga was fairly reliable. Actually, in our experience, the VR game has been the appropriate medium for conveying not just fun and the sense of being there, but also the visitors' self-awareness of their acquired knowledge. As the result, they were more likely to be curious about the settlement. Evaluating "real" learning in informal learning is not easy for novices. When considering informal learning, users do not go through a final assessment, as it is the case in formal learning methodologies. Then, we highly recommend to integrate self-assessment into some fun activities of the blended experience, for example adding mini-games with scoring, badges and leaderboards. Moreover, the use of VR games or digital activities allows to collect data in exhibitions and analyse users' behaviours automatically to improve the design of the experience. At this point, we highly recommend to define, from the very beginning, the data and the machine learning techniques to be used.

Additionally, despite visitors having liked to identify exhibition objects in the virtual space, they made mistakes. Most of miss-classifications came from Neolithic objects, such as the apple, the bow and the ladle. Users considered these objects as Non-Neolithic. We consulted with the archaeologists and they confirmed that Neolithic fruits were not mentioned neither in the physical exposition nor in the workshop. Additionally, we think that users failed with wood-made tools, such as the bow and the ladle, because they saw a lot of stone-made tools in the exposition, and also learned in the workshop how the stone was used to make tools in the Neolithic. Therefore, we suggest to strongly relate physical data in the exhibition and virtual data in the VR environment. This relationship should be two-directional. For instance, locating hints in the physical exhibition (e.g color codes in some items) could help users to achieve the challenges on the VR environment. And in reverse, balancing visual, textual and oral information from the settlement inside the virtual space could facilitate visitors' learning.

As far as the layout of the exhibition is concerned, we encourage museum managers to enable a large enough zone hosting all the learning activities. It reinforces the blended experience, letting users revisit, experiencing again, and play through the activities. Additionally, it should be noted that including VR experiences, due to their attractive and novelty appeal, nowadays could be more a distractor than an opportunity to convey information ("Guggenheim effect"), but we think that VR experiences have their role to play in museums and this role will grow in significance (especially as novelty wears off in the future). Thus, the use of these experiences in museums will be more and more commonplace and users will be more used to participate in them and, thus, decrease the Guggenheim effect. In our experience, according to the questionnaires, users felt that they learned a lot in the VR experience and became more aware about how the settlement was organised. Moreover, in informal conversations at the end of the experience, they showed interest in physically visiting La Draga. We believe that it is important for museums to start using VR activities, test their shortcomings and investigate how to overcome those. 
Design of the VR experience

Multidisciplinary works face innately contradictory constraints on design. As stated before, the project involved a team ranging from archaeologists and curators to art designers and computer scientists. Some decisions, such as the selection of virtual objects to be included in the virtual Draga and the degree of details of the environment, gave rise to lengthy discussions among team members. Archaeologists reconstructed the past based on few evidences whereas the art designers demanded a lot of details to be able to model the objects, and, at the same time, computer scientists had restrictions given by the current VR hardware to obtain interaction rates in complex environments. Although there are a lot of experiences published in the bibliography, most of them are customized for specific exhibitions. Researches such as [16] have been helpful to have a standard framework and guidelines to design virtual experiences in museums, though there is still a lot of improvement in this line.

After initial testing we learned that it is important to make some design trade-offs in order to avoid long queues in the VR exhibition. We had to strike the right balance between limiting the time of the exhibition and making sure that the amount of required learning and engagement of the users remain high. Familiarising with VR devices represents a time-consuming task and a cognitive overload for the users. On top of this, the duration of a cultural visit is usually short. Therefore, we align with previous researchers [27] and agree on the importance of designing museum learning environments that offer time flexibility. One possible solution is to find more natural interactions in VR, as it happened for mobile applications in museums, e.g. the scan-and-tilt proposal for obtaining relevant information about the exhibition [49]. Another solution is to use adaptive interaction techniques. That is, detect on the fly user profile and, if necessary, make some kind of interactions easier (e.g. grabs, throws with higher radius of influence). Moreover, the design could consider another interactions styles such as conversational ones (voice-activated commands) or gesture recognition.

Regarding VR analytics, our study identified three different profiles of users that played the gamified VR experience (Novice, Medium and Pro). The analysis of novice users helped us to identify design implications useful for future researches. We found four pattern behaviours in the Novices: Strugglers, Engaged, Explorers and Late-Explorers, which may be helpful for understanding possible future improvements to be taken into account. First of all, the so-named Strugglers (see Figure 10 (a)), those users that had problems interacting with VR controllers, visited a small area of the virtual Draga and had no opportunity to reach better game-performance. It confirms that problems with navigation and object manipulation techniques have an effect on game performance. Furthermore, the behaviour pattern of Late-Explorers (see Figure $10(\mathrm{~d})$ ) shows users struggled with VR controllers at the beginning and then decided to explore the environment rather than play. Our recommendation is to carefully design training tutorials, both at the initial phase of the experience (without forcing users to play from the very beginning) and, most 
importantly, training tutorials during run-time, i.e the adaptive management of training tutorials. In other words, we advocate for including tutorials at any time during the experience, as a tool that tailors to users needs of skills to effectively interact using VR controllers. However, since tutorials need additional time for non-experienced users, this recommendation could be costly in terms of experience's duration, which should be extended, and in terms of more VR installations in the exhibition, increasing the number of supporting staff.

On the other hand, another lesson learned comes from users that fitted the Novices cluster, and thus were supposed to be Strugglers (see Figure 10 (c)) but we classified them as Explorers. Their low game-performance was not due to problems with VR controllers but due to their desire of exploring rather than playing. In the context of cultural heritage dissemination, we suggest to offer gamified activities to users once they have already explored the environment. To play or to continue exploring the virtual space should be a decision of the visitor.

Regarding Engaged users (see Figure 10 (b)), those that spent their time interacting with fun and interactive content (e.g. pig, human). To facilitate the exploration of the virtual environment, we could take advantage of their willingness to have fun to include leisure activities in strategic regions near to items to be discovered. Indeed, our results indicate that the majority of users were not able to find all the Neolithic and Non-neolithic objects distributed along the space (the average of found objects was 4,35 out of a total of 16 objects). It could be due to time constraints or lack of situational awareness. When the reason is the latter, we recommend the use of minimaps, which show user position, relevant places in the virtual environment, and visited and un-visited zones. Moreover, the regions with leisure activities should appear highlighted in a minimap, which is a bird-eye-view of the virtual space. Undoubtedly, this kind of guide helps visitors to better explore the space.

\section{Conclusions and future work}

This research work focused on the dissemination of knowledge of La Draga Neolithic settlement. To do so, we designed a blended learning experience, which included a museum exposition, a workshop, a $360^{\circ}$ video, and a gamified VR experience. We evaluated several dimensions of the user experience (learning, realism and engagement). Results are encouraging because users highly rated the overall blended learning experience.

Additionally, we aimed to study behaviour patterns of users while immersed within the Virtual Draga. Thus, the gamified VR was made available to the general public as well as to attendants of different travelling exhibitions. The analysis of data gathered through logs yielded interesting results. Users were classified in three groups, the Novices, Medium, and Pro. The Novices, the ones that potentially had more problems, deserved more exploration. We found four patterns in Novices' behaviour: Strugglers, Engaged, Explorer, Late-Explorer. 
Interestingly, only the $23,6 \%$ of them (the Strugglers) really represented users that experienced problems. This fact has design implications that relate with the facilitation of VR skills to them but also the design of experiences that consider the Explorer profile $(60 \%)$ of users visiting museum and cultural exhibitions.

As future work we plan to extend our virtual scenario adding other virtual objects of the settlement, as well as interactions with Neolithic inhabitants to learn more about their customs (related to cook, manipulate objects, hunt animals, etc.). Additionally, we plan to improve VR interaction aspects, including a minimap, adaptive tutorials, and using multimodal interactions.

\section{References}

1. Livingstone, D. W. (2006). Informal learning: Conceptual distinctions and preliminary findings. Counterpoints, 249, 203-227.

2. Falk, J. H., and Dierking, L. D. (2000). Learning from Museums: Visitor Experiences and the Making of Meaning. Rowman \& Littlefield.

3. Schugurensky, D. (2000). The forms of informal learning: Towards a conceptualization of the field.

4. Valiathan, P. (2002). Blended learning models. Learning circuits, 3(8), 50-59.

5. Aldrich, C. (2005). Learning by doing: A comprehensive guide to simulations, computer games, and pedagogy in e-learning and other educational experiences. John Wiley \& Sons.

6. Moss, J. M. (2018). The Educational Experience of Virtual Reality: An Archaeological Case Study of the Maya Site, Vista Alegre.

7. Pujol, L. (2010). Virtual Reality as a Learning Tool for Archaeological Museums.

8. . Carrozzino, M., Lorenzini, C., Evangelista, C., Tecchia, F., and Bergamasco, M. (2015, September). AMICA: Virtual Reality as a tool for learning and communicating the craftsmanship of engraving. In Digital Heritage, 2015 (Vol. 2, pp. 187-188). IEEE.

9. Tate Modern Museum - Retrieved June 2019, https://www.tate.org.uk/whats-on/ tate-modern/exhibition/modigliani/modigliani-vr-ochre-atelier

10. Home, M. W. (2016, April). Virtual reality at the British Museum: What is the value of virtual reality environments for learning by children and young people, schools, and families. In Proceedings of the Annual Conference of Museums and the Web, Los Angeles, CA, USA (pp. 6-9).

11. National Research Council. (1995). Virtual reality: Scientific and technological challenges. National Academies Press.

12. Terradas, X., Piqué, R., Palomo, A., Antolín, F., López, O., Revelles, J., and Buxó, R. (2017). Farming practices in the early Neolithic according to agricultural tools: evidence from la Draga site (Northeastern Iberia). In Times of Neolithic Transition along the Western Mediterranean (pp. 199-220). Springer, Cham.

13. Shah, N. F. M. N., and Ghazali, M. (2018, August). A Systematic Review on Digital Technology for Enhancing User Experience in Museums. In International Conference on User Science and Engineering (pp. 35-46). Springer, Singapore.

14. Roussou, M. (2001). Immersive interactive virtual reality in the museum. Proc. of TiLE (Trends in Leisure Entertainment).

15. Bogdanovych, A., Rodríguez-Aguilar, J. A., Simoff, S., and Cohen, A. (2010). Authentic interactive reenactment of cultural heritage with $3 \mathrm{D}$ virtual worlds and artificial intelligence. Applied Artificial Intelligence, 24(6), 617-647.

16. Carrozzino, M., Colombo, M., Tecchia, F., Evangelista, C., and Bergamasco, M. (2018, June). Comparing Different Storytelling Approaches for Virtual Guides in Digital Immersive Museums. In International Conference on Augmented Reality, Virtual Reality and Computer Graphics (pp. 292-302). Springer, Cham. 
17. Miyashita, T., Meier, P., Tachikawa, T., Orlic, S., Eble, T., Scholz, V., ... and Lieberknecht, S. (2008, September). An augmented reality museum guide. In Proceedings of the 7th IEEE/ACM International Symposium on Mixed and Augmented Reality (pp. 103-106). IEEE Computer Society.

18. Hall, T., Ciolfi, L., Bannon, L., Fraser, M., Benford, S., Bowers, J., ... and Flintham, M. (2001, November). The visitor as virtual archaeologist: explorations in mixed reality technology to enhance educational and social interaction in the museum. In Proceedings of the 2001 conference on Virtual reality, archeology, and cultural heritage (pp. 91-96). ACM.

19. Wojciechowski, R., Walczak, K., White, M., and Cellary, W. (2004, April). Building virtual and augmented reality museum exhibitions. In Proceedings of the ninth international conference on 3D Web technology (pp. 135-144). ACM.

20. Gaitatzes, A., Christopoulos, D., and Roussou, M. (2001). Reviving the past: cultural heritage meets virtual reality, in Proceedings of the 2001 Conference on Virtual Reality, Archeology, and Cultural Heritage (Athens: ACM), pp. 103-110.

21. The Virtual Dutch Men - Retrieved June 2019, http://www.virtualdutchmen.com/ cases/\#entry

22. ChroniclesVR - Retrieved June 2019, http://www.chronicles-vr.co.uk/

23. Kersten, TP., Büyüksalih, G., Tschirschwitz, F., Kan, T., Deggim, S., Kaya, Y. \& Baskaraca, AP. (2017) The Selimiye Mosque of Edirne, Turkey - An Immersive and Interactive Virtual Reality Experience using HTC VIVE.International Archives of the Photogrammetry, Remote Sensing \& Spatial Information Sciences, volume 42.

24. Schofield, Guy, et al. "Viking VR: Designing a virtual reality experience for a museum." Proceedings of the 2018 Designing Interactive Systems Conference. ACM, 2018.

25. Chang, H. L., Shih, Y. C., Wang, K., Tsaih, R. H., and Lin, Z. (2018). Using Virtual Reality for Museum Exhibitions: The Effects of Attention and Engagement for National Palace Museum.

26. Carrozzino, M., \& Bergamasco, M. (2010). Beyond virtual museums: Experiencing immersive virtual reality in real museums. Journal of Cultural Heritage, 11(4), 452-458.

27. Hou, H. T., Sheng-Yi, W., Peng-Chun, L., Yao-Ting, S., Lin, J. W., and Chang, K. E. (2014). A blended mobile learning environment for museum learning. Journal of Educational Technology \& Society, 17(2).

28. Döpker, A., Brockmann, T., Stieglitz, S., and Campus, L. (2013). Use Cases for Gamification in Virtual Museums. In GI-Jahrestagung (pp. 2308-2320).

29. Liu, S., and Idris, M. Z. (2018). Constructing a framework of user experience for museum based on gamification and service design. In MATEC Web of Conferences (Vol. 176, p. 04007). EDP Sciences.

30. Terrenghi, L., and Zimmermann, A. (2004, January). Tailored audio augmented environments for museums. In Proceedings of the 9th international conference on Intelligent user interfaces (pp. 334-336). ACM.

31. Aroyo, L., Brussee, R., Rutledge, L., Gorgels, P., Stash, N., Wang, Y., ... and Bearman, D. (2007). Personalized museum experience: The Rijksmuseum use case.

32. Antoniou, A., and Lepouras, G. (2010). Modeling visitors' profiles: A study to investigate adaptation aspects for museum learning technologies. Journal on Computing and Cultural Heritage (JOCCH), 3(2), 7.

33. Sifa, R., Drachen, A., and Bauckhage, C. (2018). Profiling in Games: Understanding Behavior from Telemetry. Social Interactions in Virtual Worlds: An Interdisciplinary Perspective.

34. Bartle, R. (1996). Hearts, clubs, diamonds, spades: Players who suit MUDs. Journal of MUD research, 1(1), 19.

35. Bauckhage, C., Drachen, A., and Sifa, R. (2015). Clustering game behavior data. IEEE Transactions on Computational Intelligence and AI in Games, 7(3), 266-278.

36. Spronck, P., Balemans, I., and Van Lankveld, G. (2012, October). Player Profiling with Fallout 3. In AIIDE.

37. Antolín, F. (2014). Of cereals, poppy, acorns and hazelnuts plant economy among early farmers (5500-2300 cal BC) in the NE of the Iberian Peninsula: an archaeobotanical approach. 
38. Antolín, F., Buxó, R., Jacomet, S., Navarrete, V., \& Saña, M. (2014). An integrated perspective on farming in the early Neolithic lakeshore site of La Draga (Banyoles, Spain). Environmental archaeology, 19(3), 241-255.

39. Ferme, L. C., \& i Huerta, R. P. (2014). Landscape and forest exploitation at the ancient Neolithic site of La Draga (Banyoles, Spain). The Holocene, 24(3), 266.

40. Bosch, A., Chinchilla, J., \& Tarrús, J. (2000). El poblat lacustre neolític de La Draga. Excavacions de 1990 a 1998. Monografías del CASC, 2.

41. López i Bultó, J. O. Doctoral dissertation. (2015). Processos d'obtenció, transformació i ús de la fusta en l'assentament neolític antic de la Draga (5320-4800 cal BC).

42. Campana, I. (2018) Prehistoric house and 3D Reconstruction: Towards a BIM Archaeology. Doctoral dissertation. Bellaterra, Universitat Autònoma de Barcelona.

43. Poveda, M. O. (2015). Aprofitament i transformació de matèries primeres per a l'elaboració d'ornaments durant la prehistòria recent (5600-3400 cal. ane) al nord-est de la península Ibèrica (Doctoral dissertation, Universitat Autònoma de Barcelona).

44. Sanchez-Cabrero, R., Barrientos-Fernández, A., Arigita-García, A., Mañoso-Pacheco, L., \& Costa-Román, O. (2018). Demographic data, habits of use and personal impression of the first generation of users of virtual reality viewers in Spain. Data in brief, 21, 26512657.

45. Whittaker, J. C. (1994). Flintknapping: making and understanding stone tools. University of Texas Press.

46. Norman, D. A. (2004). Emotional design. Ubiquity, 2004(January), 1.

47. Wu, X., Kumar, V., Quinlan, J. R., Ghosh, J., Yang, Q., Motoda, H., ... \& Zhou, Z. H. (2008). Top 10 algorithms in data mining. Knowledge and information systems, 14(1), 1-37.

48. King, B., and Lord, B. (Eds.). (2015). The manual of museum learning. Rowman \& Littlefield.

49. Mantyjarvi, J., Paternò, F., Salvador, Z., \& Santoro, C. (2006, September). Scan and tilt: towards natural interaction for mobile museum guides. In Proceedings of the 8 th conference on Human-computer interaction with mobile devices and services (pp. 191194). ACM. 